\title{
Neutron Diffraction Studies of $\mathrm{NdNi}_{5} \mathrm{Sn}$ Compound
}

\author{
B. Penc ${ }^{a}$, S. Baran ${ }^{a}$, A. Hoser ${ }^{b}$, L.P. RomakA And A. Szytula ${ }^{a}$ \\ ${ }^{a}$ M. Smoluchowski Institute of Physics, Jagiellonian University, W.S. Reymonta 4, 30-059 Kraków, Poland \\ ${ }^{b}$ Helmholtz-Zentrum Berlin für Materialen und Energie GmbH, Hahn-Meitner Pl. 1, D-14 109 Berlin, Germany \\ ${ }^{c}$ Department of Inorganic Chemistry, Lviv National University, Kyryla and Mephodiy 6, 79005 Lviv, Ukraine
}

The neutron powder diffraction measurements of the $\mathrm{NdNi}_{5} \mathrm{Sn}$ compound have been performed. The obtained results indicate that this compound crystallizes in a hexagonal $\mathrm{CeNi}_{5} \mathrm{Sn}$-type crystal structure described by the space group $P 6_{3} / \mathrm{mmc}$. The parameters of the crystal structure at 1.55 and $14.8 \mathrm{~K}$ are determined. In contradiction to the magnetic data the long-range magnetic ordering was not detected up to $1.55 \mathrm{~K}$.

DOI: $10.12693 /$ APhysPolA.126.772

PACS: $61.05 . \mathrm{F}-$, 75.20.En

\section{Introduction}

The magnetic properties of $\mathrm{RNi}_{5} \mathrm{Sn}$ compounds are the object of our investigations. These compounds for $\mathrm{R}=\mathrm{La}-\mathrm{Nd}$ crystallize in a hexagonal crystal structure (space group $P 6_{3} / m m c$ ) with a complex crystal structure [1]. Preliminary magnetic measurements performed in the temperature range $80-400 \mathrm{~K}$ indicate paramagnetic properties [2]. New ac and dc magnetic data indicate for $\mathrm{NdNi}_{5} \mathrm{Sn}$ below the Néel temperature equal to $8.8 \mathrm{~K}$ - the complex magnetic properties [3]. Presented in Ref. [3] data suggest the neutron diffraction experiment for determining the magnetic structure of this compound. This work reports the results of these investigations at low temperatures.

\section{Experimental data and results}

The experiment is performed on the sample which preparation and X-ray analysis is descripted in Ref. [3]. The neutron diffractograms were obtained at temperature equal to 1.55 and $14.8 \mathrm{~K}$ with use of the E6 diffractometer at BERII reactor (Helmholtz-Zentrum Berlin). The incident neutron wavelength was $2.447 \AA$. The data were analyzed using the Rietveld-type program FullProf [4].

The neutron diffraction pattern measured at $1.55 \mathrm{~K}$ is shown in Fig. 1. Similar neutron pattern is observed at $14.8 \mathrm{~K}$. Analysis of these data confirm that the investigated compound crystallizes in a hexagonal crystal structure (space group $P 6_{3} / m m c$ ) with the following distribution of atoms: $\mathrm{Nd}_{1}$ in $2 c$ site: $1 / 3,2 / 3,1 / 4 ; \mathrm{Nd}_{2}$ in $2 a$ site: $0,0,0 ; \mathrm{Ni}$ atoms occupy four sublattices: $\mathrm{Ni}_{1}$ in $2 b$ site: $0,0,1 / 4 ; \mathrm{Ni}_{2}$ in $2 d$ site: $1 / 3,2 / 3,3 / 4 ; \mathrm{Ni}_{3}$ in $4 f$ site: $1 / 3,2 / 3, z_{1} ; \mathrm{Ni}_{4}$ in $12 k$ site: $x, y, z_{2}$ and $\mathrm{Sn}$ atoms in $4 f$ site: $1 / 3,2 / 3, z_{3}$. The determined crystal structure parameters are collected in Table. These parameters agree well with the published previously data $[1,3]$. Comparison of the data from $1.55 \mathrm{~K}$ with those from $14.8 \mathrm{~K}$ does not give evidence for the additional peaks connected with magnetic origin (see lower part in Fig. 1). This result suggests the absence of the long range magnetic order and is in contradiction with the previous magnetic

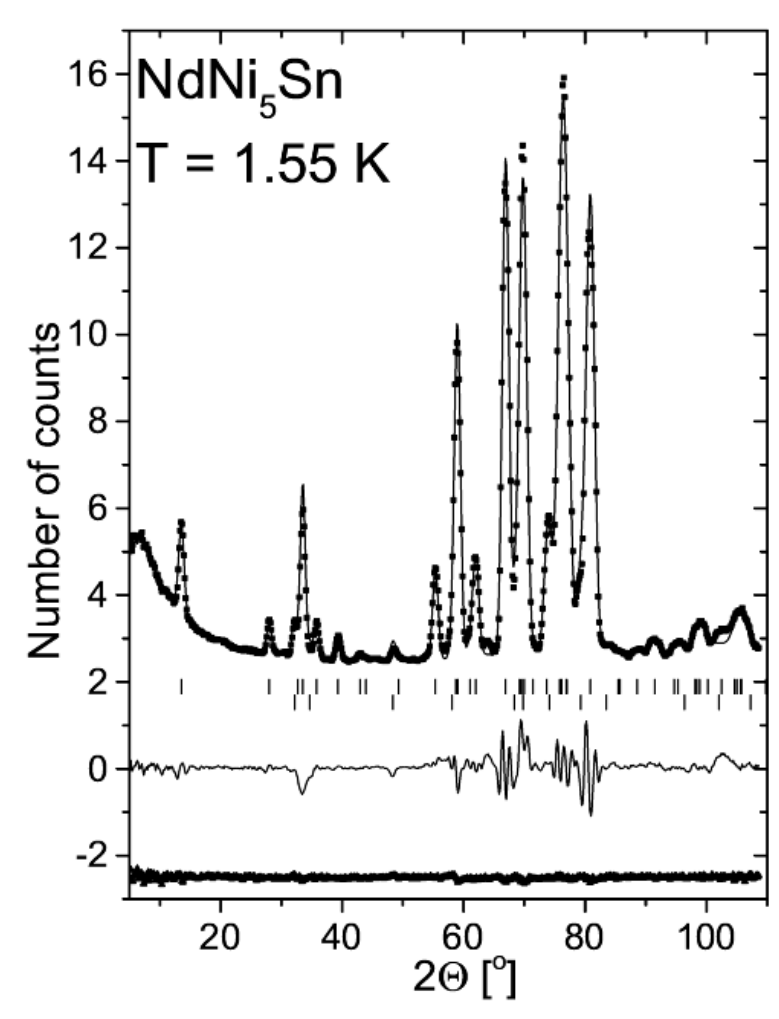

Fig. 1. Comparison between the observed and calculated neutron diffraction pattern by the Rietveld method at $1.55 \mathrm{~K}$. The symbols represent the experimental data while the solid line denotes the calculated profile. The difference between the observed and calculated intensities is shown at the bottom of diagram. The vertical bars indicate the positions of the Bragg peaks for $\mathrm{NdNi}_{5} \mathrm{Sn}$ (first row) and $\mathrm{NdNi}_{5}$ (second row). In the lower part the difference between patterns collected in 1.55 and $14.8 \mathrm{~K}$ is presented.

data [3]. These data in temperature dependence of the $\chi^{\prime}$ and $\chi^{\prime \prime}$ components of ac magnetic susceptibility give the anomalies at $8.8 \mathrm{~K}$ (see Fig. 3 in Ref. [3]) and the small ferromagnetic component with the small hysteresis in the magnetization curve (see inset in Fig. 2b in Ref. [3]). 
TABLE

Crystal and structure parameters of $\mathrm{NdNi}_{5} \mathrm{Sn}$ compounds determined from the neutron diffraction data at 1.55 and $14.8 \mathrm{~K}$.

\begin{tabular}{l|c|c}
\hline \hline Parameter & $T=1.55 \mathrm{~K}$ & $T=14.8 \mathrm{~K}$ \\
\hline$a[\AA]$ & $4.9111(11)$ & $4.9111(12)$ \\
$c[\AA]$ & $19.7524(53)$ & $19.7525(55)$ \\
$V\left[\AA^{3}\right]$ & $412.58(28)$ & $412.58(30)$ \\
$\mathrm{Ni}_{3} z_{1}$ & $0.5430(3)$ & $0.5430(3)$ \\
$\mathrm{Ni}_{4} x$ & $0.8310(3)$ & $0.8310(3)$ \\
$y$ & $0.6625(3)$ & $0.6625(2)$ \\
$z_{2}$ & $0.1459(2)$ & $0.1460(2)$ \\
$\mathrm{Sn} z_{3}$ & $0.0865(5)$ & $0.0864(6)$ \\
$R_{\text {Bragg }}[\%]$ & 4.92 & 5.07 \\
$R_{\mathrm{F}}[\%]$ & 3.44 & 3.62
\end{tabular}

Observed anomalies are probably connected with the impurity phase $\mathrm{NdNi}_{5}$. This compound is ferromagnet with the Curie temperature $7 \mathrm{~K}$ [5]. The existence of this phase is not observed in the X-ray experiment while in the neutron diffraction patterns the small intensity peak at $2 \theta=48.5^{\circ}$ indicate nearly $4 \%$ impurity phase $\mathrm{NdNi}_{5}$ which crystallizes in a hexagonal $\mathrm{CaCu}_{5-}$ type crystal structure (space group $P 6 / \mathrm{mmm}$ ) with the Nd atoms in $1 a$ site: $0,0,0$ and $\mathrm{Ni}$ atoms in $2 c: 1 / 3$, $2 / 3,0$ and $3 g: 1 / 2,0,1 / 2$ sites. Numerical analysis of our data confirm this structure with the lattice parameters $a=4.9866(23) \AA$ and $c=4.0259(17) \AA$ and $R_{\text {Bragg }}=10.4 \%$ and $R_{\mathrm{F}}=6.7 \%$.

Crystal chemical analysis [6] shown that the crystal structure of the $\mathrm{NdNi}_{5} \mathrm{Sn}$ compound can be considered as consisting of two kinds of layers, with stoichiometry $\mathrm{NdNi}_{5}$ and $\mathrm{NdNi}_{5} \mathrm{Sn}_{2}$, which stack alternately along [001] of the hexagonal unit cell (see Fig. 2 in Ref. [6]) with the sequence 1:1. In $\mathrm{NdNi}_{5} \mathrm{Sn}$ compound the $\mathrm{Nd}$ and $\mathrm{Ni}$ atoms form a close packing site. During thermal treatment in the part of sample the impurity phase $\mathrm{NdNi}_{5}$ is pushed out.

Presented results confirm that up to $1.55 \mathrm{~K}$ the long range order does not exist.

\section{Acknowledgments}

This project has been supported by the European Commission under the 7th Framework Programme through the "Research Infrastructure" action of the "Capacities" programme, NM13-II grant number 283883.

\section{References}

[1] R.V. Skolozdra, V.M. Mandzyk, L.G. Akselrud, Kristallografiya 26, 480 (1981).

[2] R.V. Skolozdra, L.P. Komarovskaya, Dokl. Akad. Nauk Ukr. SSR, Ser. A 6, 83 (1982).

[3] Yu. Tyvanchuk, L.P. Romaka, A. Szytuła, R. Duraj, A. Zarzycki, Acta Phys. Pol. A 123, 145 (2013).

[4] J. Rodriguez-Carvajal, Physica B 192, 55 (1993).

[5] V.M.T.S. Barthem, D. Gignoux, A. Nait-Saada, D. Schmitt, A.Y. Takeuchi, J. Magn. Magn. Mater. 80, 142 (1989).

[6] M. Sato, V.A. Yartys, J. Alloys Comp. 379, 171 (2004). 